\title{
Identification of $C R B 1$ mutations in two Chinese consanguineous families exhibiting autosomal recessive retinitis pigmentosa
}

\author{
XIAOXIN GUO ${ }^{1 *}$, JIE LI $^{2 *}$, QINGWEI WANG ${ }^{1,3^{*}}$, YI SHU $^{1}$, JIN WANG $^{1}$, LIJIA CHEN $^{4}$, HOUBIN ZHANG ${ }^{1}$,

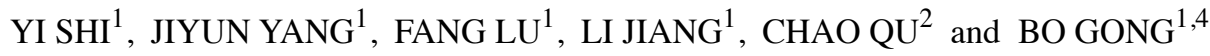 \\ ${ }^{1}$ Key Laboratory for Human Disease Gene Study of Sichuan Province and Department of Laboratory Medicine, \\ ${ }^{2}$ Department of Ophthalmology, Sichuan Academy of Medical Sciences and Sichuan Provincial People's Hospital, \\ School of Medicine, University of Electronic Science and Technology of China, Chengdu, Sichuan 610072; \\ ${ }^{3}$ Institute of Chengdu Biology, Sichuan Translational Medicine Hospital, Chinese Academy of Sciences, \\ Chengdu, Sichuan 610000; ${ }^{4}$ Department of Ophthalmology and Visual Sciences, \\ The Chinese University of Hong Kong, Hong Kong, SAR, P.R. China
}

Received January 24, 2019; Accepted June 12, 2019

DOI: $10.3892 / \mathrm{mmr} .2019 .10495$

\begin{abstract}
Retinitis pigmentosa (RP) is a leading cause of inherited blindness characterized by progressive loss of retinal photoreceptor cells. The present study aimed to identify the causative gene mutations in two Chinese families with autosomal recessive retinitis pigmentosa (arRP). Two Chinese consanguineous arRP families (RP-2284 and RP-2360) were recruited in this study, involving totally three affected and 25 unaffected members. All the affected members underwent a complete ophthalmic examination, including fundus photography, multifocal electroretinography (ERG) and full field ERG. Exome sequencing was performed on the three RP patients in the two families, followed by direct Sanger sequencing in all the family members and in 1,260 unrelated controls for validation of the mutations identified. Two homozygous missense mutations in the crumbs homolog $1(C R B 1)$ gene, which is known to cause severe retinal dystrophies, were found
\end{abstract}

Correspondence to: Dr Bo Gong, Key Laboratory for Human Disease Gene Study of Sichuan Province and Department of Laboratory Medicine, Sichuan Academy of Medical Sciences and Sichuan Provincial People's Hospital, School of Medicine, University of Electronic Science and Technology of China, 32 Road West 2, The First Ring, Chengdu, Sichuan 610072, P.R. China E-mail: gongbo2007@hotmail.com

Dr Chao Qu, Department of Ophthalmology, Sichuan Academy of Medical Sciences and Sichuan Provincial People's Hospital, School of Medicine, University of Electronic Science and Technology of China, 32 Road West 2, The First Ring, Chengdu, Sichuan 610072, P.R. China

E-mail: lucyjeffersonqu@hotmail.com

*Contributed equally

Key words: autosomal recessive retinitis pigmentosa, crumbs homolog 1 gene, $C R B 1$, homozygous mutation, exome sequencing to be related to the phenotype of the two arRP families. The homozygous missense mutation c.1997 T>A in CRBI was detected in two patients in the RP-2284 family. The proband in the RP-2360 family was the only RP patient and was found to carry the novel homozygous missense mutation c.2426 A $>\mathrm{C}$ in $C R B 1$. The two mutations were heterozygous or absent in the other healthy family members, and they were absent in the 1,260 controls. The amino acid changes in the CRB1 protein affected by the two mutations were predicted to be damaging by Polyohen-2. Our study reported two CRB1 mutations causing arRP in two Chinese families, which expands the $C R B 1$ mutation spectrum of RP in the Chinese population and emphasizes the causative role of $C R B 1$ in RP.

\section{Introduction}

Retinitis pigmentosa (RP) is caused by a progressive degeneration of photoreceptor cells that initially primarily affect the rods whereas the function of the cones is compromised as the disease progresses (1). Due to its heterogeneity and diversity of inheritance patterns, the clinical presentation of RP is variable. Some RP patients lose vision in their childhood, whereas other patients retain normal vision until mid-adulthood. The clinical symptoms of RP include night blindness and gradual loss of the peripheral visual field caused by rod photoreceptor lost. Later with the loss of cone photoreceptors, the central vision is impaired and patients may ultimately suffer from legal blindness ultimately (1). The worldwide prevalence of $\mathrm{RP}$ is approximately 1 in 4,000 , or more than 1 million individuals (2).

RP is clinically and genetically heterogeneous. It can be inherited as an autosomal dominant (30-40\%), autosomal recessive (50-60\%), or X-linked (5-15\%) trait (3). Some patients who have no known family history are classified as simplex RP. Autosomal recessive RP (arRP) is the most common form of RP worldwide. To date, more than 80 genes (https://sph.uth.edu/retnet/) have been implicated in syndromic and non-syndromic forms of RP. However, these identified 
genes account for no more than $60 \%$ of RP cases, and there has been limited success in screening known genes for RP using conventional Sanger sequencing. Recently, exome sequencing has been successfully used to identify genes associated with Mendelian disorders $(4,5)$. Coupled with DNA capture technology, the next-generation sequencing (NGS) platform enables rapid and cost-effective parallel sequencing of a large panel of disease genes. In some recent studies, NGS has provided a promising alternative for the molecular diagnosis and gene identification of RP (6-10).

In the present study, the identification of disease-causing mutations of RP was presented by using exome sequencing in two Chinese families with arRP. This report concerns the clinical features and genetic findings of the two arRP families.

\section{Patients and methods}

Study subjects. Two arRP pedigrees with Chinese origin were recruited from Sichuan Provincial People's Hospital, including a four-generation family with two consanguineous arRP patients and 16 unaffected members (RP-2284; Fig. 1A), and a four-generation family with one consanguineous RP patient and nine unaffected members (RP-2360; Fig. 1B). In addition, 1,260 unrelated healthy individuals of Chinese Han origin were also recruited. This study was approved by the Institutional Review Board of the Sichuan Provincial People's Hospital, University of Electronic Science and Technology of China. Informed written consent was obtained from each individual prior to participation in this study. All procedures were carried out in accordance with the tenets of the Declaration of Helsinki.

Clinical diagnosis. The clinical information of the two families is listed in Table I. Each family member underwent a complete ophthalmic examination, including best-corrected visual acuity (BCVA), slit-lamp biomicroscopy, fundus photography if possible, visual field testing (Octopus; Interzeag AG) and electroretinography (ERG). ERGs were performed using a multifocal ERG recorder (GT-2008V-IV; Chongqing Kanghua Ruiming S\&T Co., Ltd) and corneal contact lens electrodes. The ERG protocol complied with the standards of the International Society for Clinical Electrophysiology of Vision. Diagnosis of RP in the three patients was based on poor night vision at an early age, followed by progressive loss of peripheral visual fields, and extinguished ERG responses, along with abnormal fundus changes (2). In contrast, all the unaffected family members, and the unrelated control individuals had no signs of retinal diseases.

DNA extraction. Genomic DNA were extracted from the peripheral blood of all family members (RP-2284 and RP-2360) and 1,260 controls, using a TIANamp Blood DNA kit (cat. no. DP348) according to the manufacturer's protocol (Tiangen Biotech Co., Ltd.).

Exome sequencing and variant detection. Exome sequencing was performed on patients IV:2 and IV:4 in RP-2284 and patient IV:3 in RP-2360 by Genergy Biotechnology, Co., Ltd. The sequenced samples were prepared according to the Illumina protocols of the SureSelect Target Enrichment
System Capture Process (Illumina, Inc.) and exome sequencing analysis was performed as described previously (11). Briefly, the reads were mapped against UCSC hg19 (http://genome.ucsc.edu/) by BWA (http://bio-bwa. sourceforge.net/). The SNPs and Indels were detected by SAMTOOLS (http://samtools.sourceforge.net/). The detected variants were annotated and filtered based on public and in-house databases, under the following conditions: i) variants within intergenic, intronic, and UTR regions and synonymous mutations were excluded from downstream analysis; ii) variants in dbSNP138 (http://www.ncbi.nlm.nih.gov/projects/SNP/), 1000 Genome project (ftp://ftp.1000genomes.ebi. ac.uk/voll/ftp), Exome Aggregation Consortium, YH Database (http://yh.genomics.org.cn/), the HapMap Project (ftp://ftp. ncbi.nlm.nih.gov/hapmap) and our in-house database generated from 2,010 samples of exome sequencing, were excluded; iii) Polyphen 2 (http://genetics.bwh. harvard.edu/pph2/) was used to predict the possible damaging impacts of each mutation.

Mutation validation. The CRB1 mutations identified by exome sequencing were further confirmed in all members of the two families and in 1,260 controls by direct Sanger sequencing, using the PCR primers (CRB1-c.1997T >A-F, 5'-AGTGGCATT TCGTGGAGGTA-3' and $C R B 1-\mathrm{c} .1997 \mathrm{~T}>\mathrm{A}-\mathrm{R}:$ 5'-GCTGTT $^{\prime}$ TCTGCTCTGCTCTG-3'; CRB1-c.2426A>C-F: 5'-TTTGTC CGAACGCTTCAACC-3' and CRB1-c.2426A>C-R: 5'-TCT TGCTTGTCAGGTAGGCC-3') designed by Primer 3.0 (http://primer3.ut.ee/) and synthesized by Invitrogen (Thermo Fisher Scientific, Inc.). Direct sequencing was performed in an ABI 3130XL genetic analyzer according to ABI BigDye sequencing protocols (Thermo Fisher Scientific, Inc.).

\section{Results}

Patients and clinical information. Two Chinese consanguineous arRP families (RP-2284 and RP-2360, Fig. 1) were recruited in this study. Two subjects (IV:2 and IV:4) were diagnosed with RP in family RP-2284, which exhibited a pattern of recessive inheritance. They presented typical clinical symptoms, including night blindness rapidly progressing to severe visual disability. The fundus examination of the proband (IV:2) showed pallor in the optic nerve head, retinal artery attenuation, and mid-peripheral defuse and dense bone-spicule pigmentation (Fig. 2A and B). Another affected member (IV:4) had similar clinical manifestations to the proband and presented with macular degeneration (Fig. 2C). Family RP-2360 included one RP patient (IV:3, the proband), who had experienced night blindness since 23 years of age and progressive bilateral visual loss (Table I). She presented with early-onset and markedly decreased visual acuity (OD: 20/400, OS: 20/400) in both eyes (Table I). The fundus image in the right eye was blurred due to dense posterior subcapsular cataract. Fundus examination of her left eye showed similar features to those of patient IV:2 in RP-2284 (data not shown). None of the three patients exhibited glaucoma features, and their ERG examination showed no recordable response under either scotopic or photopic condition, indicating significant loss of function in the both rods and cones (Fig. 2D and E). 
Table I. Genotypes and phenotypes of the two RP family members.

A, Family RP-2284

\begin{tabular}{|c|c|c|c|c|c|c|c|}
\hline $\begin{array}{l}\text { Family } \\
\text { member }\end{array}$ & $\begin{array}{c}\text { Age } \\
\text { (years)/sex }\end{array}$ & Phenotype & $\begin{array}{l}\text { Age at } \\
\text { onset } \\
\text { (years) }\end{array}$ & $\begin{array}{l}\text { Visual acuity } \\
\text { (OD/OS) }\end{array}$ & $\begin{array}{c}\text { Fundus } \\
\text { appearance }\end{array}$ & Mutation & $\begin{array}{l}\text { Mutation } \\
\text { type }\end{array}$ \\
\hline $\mathrm{V}: 1$ & $6 / \mathrm{F}$ & Normal & - & - & - & C.1997 T>A & Het \\
\hline IV:1 & $30 / \mathrm{F}$ & Normal & - & $1.0 / 1.0$ & Normal & No mutation & - \\
\hline IV:2 & $31 / \mathrm{M}$ & $\mathrm{RP}$ & 24 & Light perception & RVA and DBSP & C.1997 T>A & Hom \\
\hline IV:3 & 28/M & Normal & - & $1.0 / 1.0$ & Normal & C.1997 T>A & Het \\
\hline IV:4 & $23 / \mathrm{M}$ & $\mathrm{RP}$ & 22 & Counting fingers & RVA, DBSP and MD & C.1997 T>A & Hom \\
\hline IV:5 & $26 / M$ & Normal & & $1.0 / 1.0$ & Normal & No mutation & - \\
\hline III: 1 & $50 / \mathrm{M}$ & Normal & - & - & - & C.1997 T>A & Het \\
\hline III:2 & $52 / \mathrm{F}$ & Normal & - & $1.0 / 0.9$ & Normal & No mutation & - \\
\hline III:3 & $54 / \mathrm{F}$ & Normal & - & $1.0 / 1.0$ & Normal & C. $1997 \mathrm{~T}>\mathrm{A}$ & Het \\
\hline III:4 & $55 / \mathrm{M}$ & Normal & - & $1.0 / 0.8$ & Normal & C. $1997 \mathrm{~T}>\mathrm{A}$ & Het \\
\hline III:5 & $53 / \mathrm{F}$ & Normal & - & - & - & C.1997 T>A & Het \\
\hline III:6 & $50 / \mathrm{F}$ & Normal & - & $0.8 / 0.8$ & Normal & No mutation & - \\
\hline III:7 & $48 / \mathrm{F}$ & Normal & - & - & - & C. $1997 \mathrm{~T}>\mathrm{A}$ & Het \\
\hline III:8 & $49 / \mathrm{F}$ & Normal & - & $0.8 / 0.8$ & Normal & No mutation & - \\
\hline III:9 & $51 / \mathrm{M}$ & Normal & - & $0.9 / 0.8$ & Normal & C.1997 T>A & Het \\
\hline III: 10 & $48 / \mathrm{F}$ & Normal & - & $0.8 / 1.0$ & Normal & C.1997 T>A & Het \\
\hline III:11 & $46 / F$ & Normal & - & - & - & No mutation & - \\
\hline II: 1 & 78/M & Cataract & - & - & - & C.1997 T>A & Het \\
\hline
\end{tabular}

B, Family RP-2360

\begin{tabular}{|c|c|c|c|c|c|c|c|}
\hline $\begin{array}{l}\text { Family } \\
\text { member }\end{array}$ & $\begin{array}{c}\text { Age } \\
\text { (years)/sex }\end{array}$ & Phenotype & $\begin{array}{c}\text { Age at } \\
\text { onset } \\
\text { (years) }\end{array}$ & $\begin{array}{l}\text { Visual acuity } \\
\text { (OD/OS) }\end{array}$ & $\begin{array}{c}\text { Fundus } \\
\text { appearance }\end{array}$ & Mutation & $\begin{array}{l}\text { Mutation } \\
\text { type }\end{array}$ \\
\hline $\mathrm{V}: 1$ & $18 / \mathrm{F}$ & Normal & - & - & Normal & C. $2426 \mathrm{~A}>\mathrm{C}$ & Het \\
\hline IV:1 & $41 / \mathrm{F}$ & Normal & - & $1.0 / 1.0$ & Normal & C. $2426 \mathrm{~A}>\mathrm{C}$ & Het \\
\hline IV:2 & $45 / M$ & Normal & - & $1.0 / 0.9$ & Normal & No mutation & - \\
\hline IV:3 & $43 / \mathrm{F}$ & $\mathrm{RP}$ & 23 & Counting fingers & OS: RVA and DBSP & C. $2426 \mathrm{~A}>\mathrm{C}$ & Hom \\
\hline IV:4 & $46 / F$ & Normal & - & - & - & No mutation & - \\
\hline III:1 & $70 / \mathrm{M}$ & Normal & - & $0.8 / 0.7$ & Normal & C. $2426 \mathrm{~A}>\mathrm{C}$ & Het \\
\hline III: 2 & $69 / \mathrm{F}$ & Normal & - & $0.7 / 0.8$ & Normal & C. $2426 \mathrm{~A}>\mathrm{C}$ & Het \\
\hline III:3 & $67 / \mathrm{F}$ & Normal & - & - & - & C. $2426 \mathrm{~A}>\mathrm{C}$ & Het \\
\hline III: 4 & $66 / \mathrm{F}$ & Normal & - & $0.8 / 0.8$ & Normal & No mutation & - \\
\hline II:1 & $92 / \mathrm{M}$ & Cataract & - & - & - & No mutation & - \\
\hline
\end{tabular}

RP, retinitis pigmentosa; Hom, homozygous; Het, heterozygous; F, female; M, male; RVA, retinal vascular attenuation; DBSP, dense bone-spicule pigmentation; MD, macular degeneration; OD, right eye; OS, left eye; -, not available.

Exome sequencing and data analysis. Exome sequencing was performed on two patients (IV:2 and IV:4) in the RP-2284 family and on one patient (IV:3) in the RP-2360 family with a mean read depth of target regions of $52.3 \mathrm{x}$. There were 18,793 SNPs in the coding regions $(8,763$ non-synonymous SNPs, 9,836 synonymous SNPs, and 873 other types of SNPs) and 387 coding indels identified. To identify the disease-causing mutations, the researchers paid close attention to the functional SNPs/indels in homozygous or compound heterozygous status including frameshift coding-region insertion or deletions and non-synonymous variants which were more likely to be pathogenic. Detected variants were compared with HapMap Project, dbSNP138, 1000 Genomes Project, Exome Aggregation Consortium, YH Database, and our in-house database. The rare homozygous variants shared by the two affected patients in RP-2284 and RP-2360 families 
A

Family RP-2284

I

$\varnothing-\square$

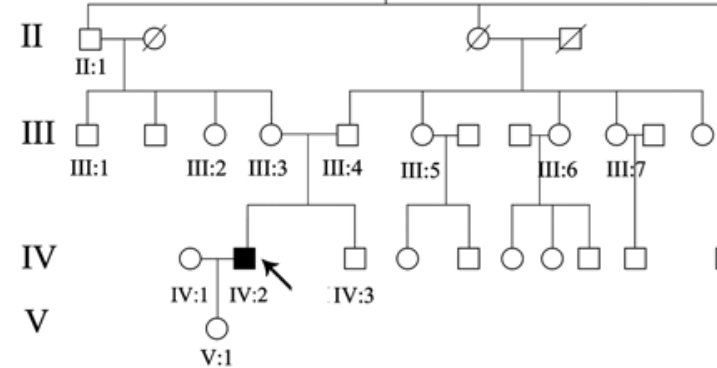

B

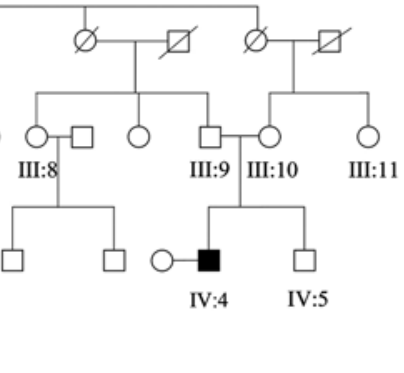

I
Family RP-2360

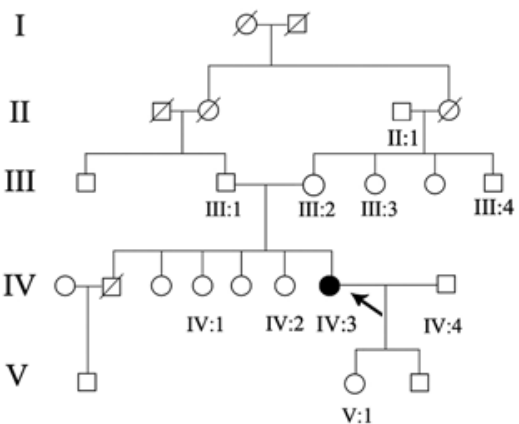

Figure 1. Pedigrees of two Chinese consanguineous families with arRP. (A and B) Two arRP families were recruited in this study. The arrow indicates the proband in each family. Affected individuals are indicated by filled symbols and open symbols indicated unaffected individuals. RP-2284, a four-generation family with two consanguineous arRP patients and 16 unaffected members; RP-2360, a four-generation family with one consanguineous RP patient and nine unaffected members are shown. arRP, autosomal recessive retinitis pigmentosa.
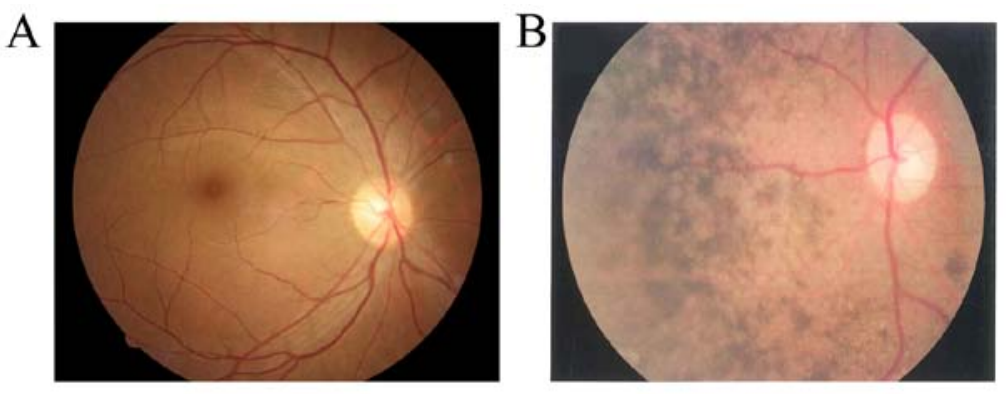

$\mathrm{D}$

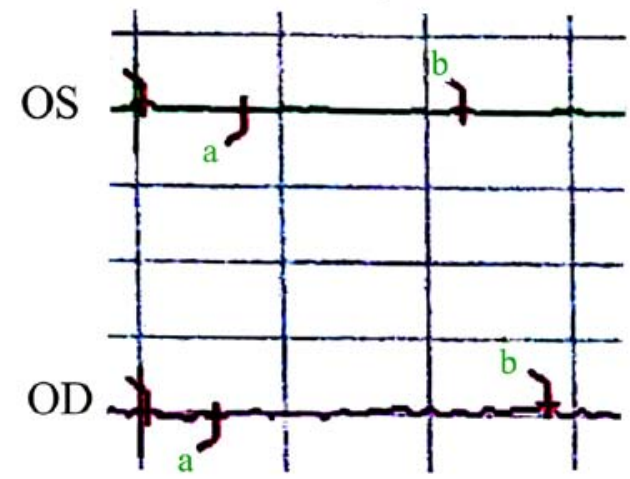

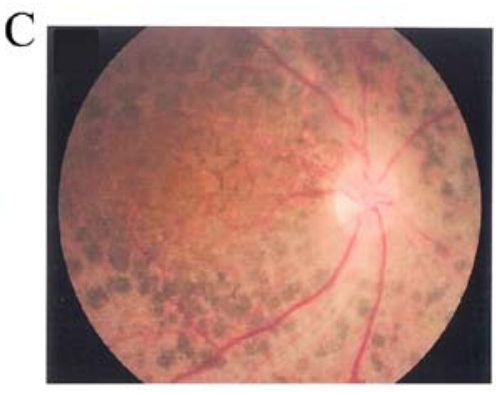

E

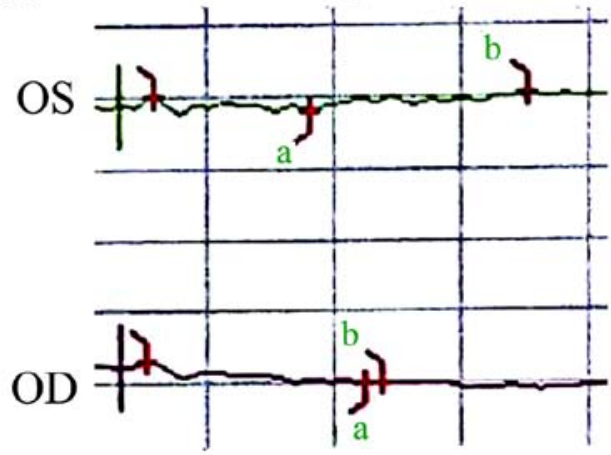

Figure 2. Representative clinical features of arRP patients. (A-C) Compared to the normal individual (IV:3), peripheral pigmentation and retinal vascular attenuation were noted in the proband's fundus photograph, as well as peripheral defuse pigmentation complicated with macular degeneration in another patient (IV:4) in family RP-2284. (D and E) ERG records showed no detectable rod and cone responses in either eye of the proband in family RP-2284. $\mathrm{OD}$, right eye; OS, left eye. arRP, autosomal recessive retinitis pigmentosa.

are listed in Table SI. Using the autosomal recessive mode, two homozygous mutations, c.1997 T>A and c.2426 A>C, were identified in the CRB1 gene (NM_201253.2) in the RP-2284 and RP-2360 families, respectively.

Mutation validation and analysis. In the RP-2284 family, the presence of homozygous mutation c.1997 T>A in two patients (IV:2 and IV:4) was confirmed by Sanger sequencing (Fig. 3B). The parents and other unaffected siblings of these two patients were either unaffected heterozygous carriers of c.1997 T>A, or had a normal $C R B I$ sequence without any mutation (Table I). In the RP-2360 family, direct Sanger sequencing confirmed the presence of homozygous mutation c.2426 A>C (Fig. 3A) in the proband (IV:3), whereas all other unaffected family members were heterozygotes or normal. The two homozygous mutations described above were absent in the 1,260 Han Chinese healthy controls. These results reveal a complete co-segregation of the two CRB1 mutations with RP within the respective families. The genotypes of the members of families RP-2284 and RP-2360 are shown in Table I.

The c.1997 T>A (p.V666D) homozygous mutation is expected to change valine (hydrophobic non-polar) to aspartic acid (hydrophilic positively charged) at codon 666 in exon 6. The substituted amino acid is predicted to alter the hydrophobicity and chargeability of the CRB1 protein. The c.2426 A>C (p.Q809T) mutation introduced a substitution 
A

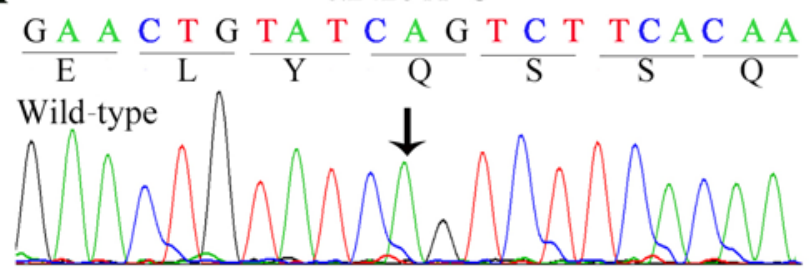

Heterozygous mutant

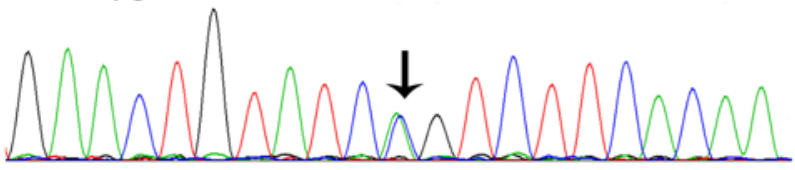

Homozygous mutant

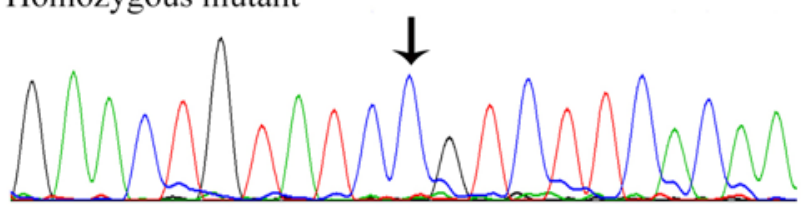

B

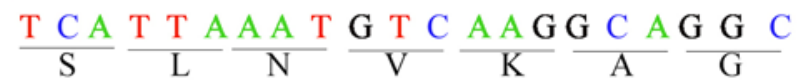

Wild-type

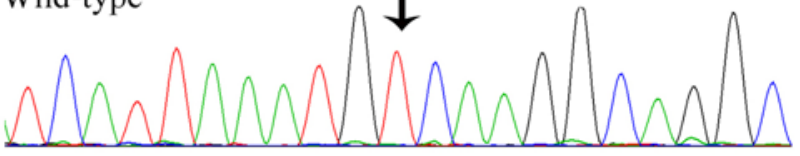

Heterozygous mutant

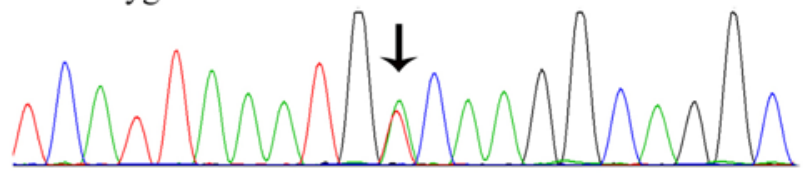

Homozygous mutant

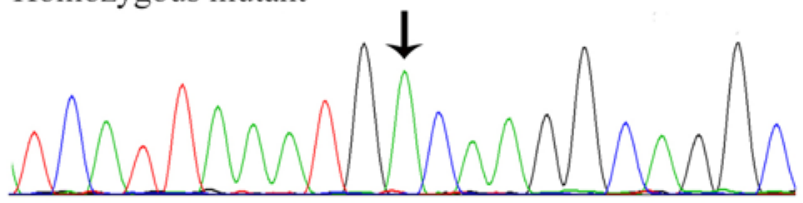

Figure 3. $C R B 1$ mutations were identified in two Chinese consanguineous arRP families. (A) A normal sequence of $C R B 1$ from an unaffected member, a heterozygous A to C substitution at codon 2,426 from the proband's parents, and a homozygous mutation from the patients were identified in family RP-2284. (B) A normal sequence of CRB1 from the proband's sister, a heterozygous T to A substitution at codon 1,997 from the proband's parents, and a homozygous mutation from the proband were identified in family RP-2236. arRP, autosomal recessive retinitis pigmentosa; $C R B 1$, crumbs homolog 1.

A
H. sapiens
P. troglodytes
M. mulatta
C. lupus
M. musculus
R. norvegicus
G. gallus
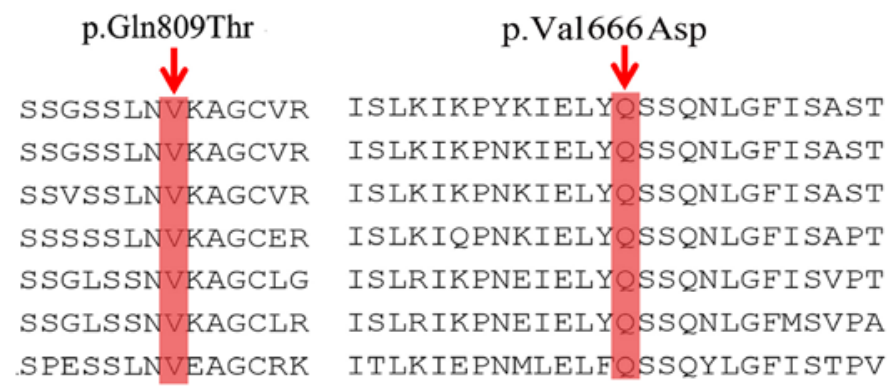

B

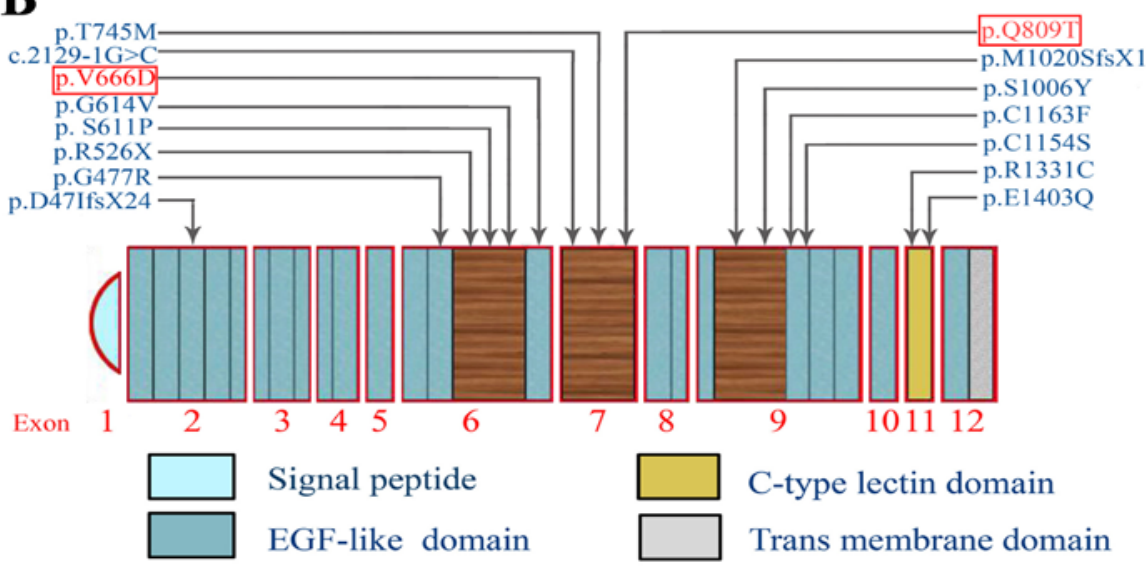

Laminin AG like domain

Figure 4. Orthologous protein sequence alignment and structure diagram of CRB1. (A) Orthologous protein sequence alignment showed the seven mutation sites occurring in a highly conserved region of CRB1 among different species. (B) Fifteen mutations that were identified in Chinese RP patients occurred mainly at exon 6,7 and 9 within a laminin A globular (AG)-like domain. The boxed mutations in red were newly identified in the present study. RP, retinitis pigmentosa; CRB1, crumbs homolog 1.

of glutamine for threonine at codon 809 in exon 7 (Fig. 4B). Comparative amino acid sequence alignment of the CRB1 proteins across human and other species revealed that the two mutations occurred at highly conserved positions (Fig. 4A). 
These changes were predicted to be damaging to CRB1 using Polyohen-2.

\section{Discussion}

A previous study demonstrated that the $C R B 1$ gene was responsible for retinitis pigmentosa (RP) at the RP12 locus (12) and more than 150 disease-associated variants in $C R B 1$ have been reported (13). Most of the mutations occur in exon 7 (27\%) and exon 9 (41\%), which encode the second and the third laminin AG-like domains, respectively (13). In the present study, two homozygous mutations c.1997 $\mathrm{T}>\mathrm{A}$ and c.2426 $\mathrm{A}>\mathrm{C}$ in $C R B 1$ causing autosomal recessive retinitis pigmentosa (arRP) in two Chinese families were identified using exome sequencing. This result expanded the $C R B 1$ mutation spectrum causing $\mathrm{RP}$, which may contribute to improvement of the molecular prognosis of RP.

Studies have reported that approximately 194 mutations in $C R B 1$ have been found to be related to the pathogenesis of arRP in diverse populations, including 189 mutations in the extracellular region (14-17). CRB1 is a human homolog of Drosophila crumbs, a well conserved gene with homologue across multiple phyla (18). It is located at 1q31.3 and consists of 12 exons spanning $210 \mathrm{~kb}$ of genomic DNA (12). This gene encodes two different proteins of 1,376 and 1,406 amino acids, and both protein are composed of three parts: 19 epidermal growth factor (EGF)-like domains, three laminin A globular (AG)-like domains and a signal peptide sequence. Beyond that, the longer isoform also contains additional transmembrane and cytoplasmatic domains (19). CRB1 is a transmembrane protein and is mainly expressed in the inner segments of mammalian photoreceptors as well as in the brain $(12,18)$. It plays an essential role in the development of the retina $(20,21)$. CRB1 is involved in photoreceptor morphogenesis in the retina (22). Mutations in CRB1 may restrain retinal development and result in a loss of photoreceptor signaling (23). In the mouse and human retina, the protein Crb1 or CRB1 is concentrated in the vicinity of the outer limiting membrane and is expressed in photoreceptor inner segments, in Muller cell and the epithelial cells (24). The Crbl mutant in mouse showed a developmental defect of retina, including a disrupted outer limiting membrane and folded retina (25).

The present study involved two Han Chinese arRP families. An analysis of the exome sequencing data revealed that only the two homozygous mutations c.1997 T>A (p.V666D) and c.2426 A>C (p.Q809T) in CRB1 were found to be co-segregated with arRP in the families. The two mutations have never been reported in the ExAC database (http://exac.broadinstitute. org/) or the human gene mutation database (http://www. hgmd.org/). The clinical findings associated with retinal dystrophies caused by $C R B 1$ mutations include congenital blindness, LCA, to early-onset rod-cone dystrophy and arRP. Mutations in CRB1 account for $10-15 \%$ of all patients with LCA and as many as $6.5 \%$ of all patients with arRP. In this study, the age of onset of patients in the two RP families was approximately 22-24 years of age. The homozygous mutation c.2426 A >C found in family RP-2360 is located at exon 7 of the $C R B 1$ gene, encoding the second laminin AG-like domain. Apart from the proband (IV:3) who carried the homozygous mutation c.2426 $\mathrm{A}>\mathrm{C}$, other family members carried either heterozygous mutation or no mutation. The proband's parents had a consanguineous marriage, and they were each heterozygous for c.2426 A>C, which was also found in members V:1, IV:1 and III:3 (Table I). As previously reported, laminin AG-like domain is predicted to affect interactions among proteins, calcium binding, and protein folding (26). This mutation introduces a substitution of glutanine to threonine, which may dramatically affect the function of the second laminin AG-like domain of the CRB1 protein (PolyPhen2 scores $\sim 0.824$ ). There were many disease causative mutations reported in the laminin AG-like domain, which indicates that this domain plays an important role in CRB1 function (24). The mutation c.1997 T>A identified in family RP-2284, was previously reported by Wang et al as present in RP patents in compound heterozygote (27), which further confirmed its pathogenic role in RP. In this pedigree with a consanguineous marriage, the proband's parents (III:3 and III:4), daughter (V:1), aunts (III:5 and III:7), uncle (III:1) and brother (IV:3), as well as another patient's parents (III:9 and III:10), were found to carry the heterozygous mutation (Table I). The change could convert valine to aspartic acid at codon 666 (p.V666 D). This substitution was predicted to be damaging by Polyohen-2 and to alter the hydrophobicity and chargeability of the CRB1 protein. Nevertheless, how these mutations exactly affect the function of CRB1 protein remains unclear. Further functional research is essential to elucidate the underlying mechanism governing how the two homozygous mutations result in RP.

In conclusion, the present study revealed two homozygous mutations c.1997 T>A (p.V 666D) and c.2426 A>C (p.Q809T) in the $C R B 1$ gene in two consanguineous Chinese families with arRP via exome sequencing. These data expand the $C R B 1$ mutation spectrum causing RP and may contribute to an improvement in the molecular diagnosis for retinal dystrophies.

\section{Acknowledgements}

Not applicable.

\section{Funding}

The present study was supported by grants from the Natural Science Foundation of China (81670853, 81700841, 81670893, 81570882 and 81670877), CAS 'Light of West China' Program (to BG), Science and Technology Department of Sichuan Province (2019JDJQ0031 and 2014FZ0124), Foundation for Technology \& Science \& Technology Bureau of Chengdu (2015-HM02-00094-SF and 2018-YF05-00348-SN).

\section{Availability of data and materials}

The datasets used and/or analyzed during the current study are available from the corresponding author on reasonable request.

\section{Authors' contributions}

BG, CQ, JL, QW and JY conceived the experiment. XG, JW and YS performed the experiments. YS, FL, JY, LJ, CQ and $\mathrm{HZ}$ recruited the patients. BG, FL, LC, QW, LJ, CQ and HZ 
analyzed the data. XG and JL drafted the manuscript. JY and $\mathrm{HZ}$ critically revised the manuscript for important intellectual content. All authors read and approved the manuscript and agreed to be accountable for all aspects of the research in ensuring that the accuracy or integrity of any part of the work are appropriately investigated and resolved.

\section{Ethics approval and consent to participate}

This study was approved by the Institutional Review Board of the Sichuan Provincial People's Hospital, University of Electronic Science and Technology of China. Informed written consent was obtained from each individual prior to participation in this study. All procedures were carried out in accordance with the tenets of the Declaration of Helsinki.

\section{Patient consent for publication}

Not applicable.

\section{Competing interests}

The authors delare that they have no competing interests.

\section{References}

1. Bird AC: Retinal photoreceptor dystrophies LI. Edward Jackson Memorial Lecture. Am J Ophthalmol 119: 543-562, 1995.

2. Hartong DT, Berson EL and Dryja TP: Retinitis pigmentosa. Lancet 368: 1795-1809, 2006.

3. Rivolta C, Sharon D, DeAngelis MM and Dryja TP: Retinitis pigmentosa and allied diseases: Numerous diseases, genes, and inheritance patterns. Hum Mol Genet 11: 1219-1227, 2002.

4. Ng SB, Turner EH, Robertson PD, Flygare SD, Bigham AW, Lee C, Shaffer T, Wong M, Bhattacharjee A, Eichler EE, et al: Targeted capture and massively parallel sequencing of 12 human exomes. Nature 461: 272-276, 2009.

5. Ng SB, Buckingham KJ, Lee C, Bigham AW, Tabor HK, Dent KM, Huff CD, Shannon PT, Jabs EW, Nickerson DA, et al: Exome sequencing identifies the cause of a mendelian disorder. Nat Genet 42: 30-35, 2010 .

6. Neveling K, Collin RW, Gilissen C, van Huet RA, Visser L, Kwint MP, Gijsen SJ, Zonneveld MN, Wieskamp N, de Ligt J, et al: Next-generation genetic testing for retinitis pigmentosa. Hum Mutat 33: 963-972, 2012.

7. O'Sullivan J, Mullaney BG, Bhaskar SS, Dickerson JE, Hall G, O'Grady A, Webster A, Ramsden SC and Black GC: A paradigm shift in the delivery of services for diagnosis of inherited retinal disease. J Med Genet 49: 322-326, 2012.

8. Katagiri S, Akahori M, Sergeev Y, Yoshitake K, Ikeo K, Furuno M, Hayashi T, Kondo M, Ueno S, Tsunoda K, et al: Whole exome analysis identifies frequent CNGA1 mutations in Japanese population with autosomal recessive retinitis pigmentosa. PLoS One 9: e108721, 2014.

9. Wu J, Chen L, Tam OS, Huang XF, Pang CP and Jin ZB: Whole exome sequencing reveals genetic predisposition in a large family with retinitis pigmentosa. Biomed Res Int 2014: 302487, 2014.

10. Villanueva A, Willer JR, Bryois J, Dermitzakis ET, Katsanis N and Davis EE: Whole exome sequencing of a dominant retinitis pigmentosa family identifies a novel deletion in PRPF31. Invest Ophthalmol Vis Sci 55: 2121-2129, 2014.

11. Zhou Y, Shuai P, Li X, Wang J, Yang Y, Hao F, Lin H, Zhang D and Gong B: Association of SOD2 polymorphisms with primary open angle glaucoma in a Chinese population. Ophthalmic Genet 36: 43-49, 2015.
12. den Hollander AI, ten Brink JB, de Kok YJ, van Soest S, van den Born LI, van Driel MA, van de Pol DJ, Payne AM, Bhattacharya SS, Kellner U, et al: Mutations in a human homologue of Drosophila crumbs cause retinitis pigmentosa (RP12). Nat Genet 23: 217-221, 1999.

13. Slavotinek AM: The family of crumbs genes and human disease. Mol Syndromol 7: 274-281, 2016.

14. Cordovez JA, Traboulsi EI, Capasso JE, Sadagopan KA, Ganesh A, Rychwalski PJ, Neely KA, Brodie SE and Levin AV: Retinal dystrophy with intraretinal cystoid spaces associated with mutations in the crumbs homologue (CRB1) gene. Ophthalmic Genet 36: 257-264, 2015.

15. Booij JC, Florijn RJ, ten Brink JB, Loves W, Meire F, van Schooneveld MJ, de Jong PT and Bergen AA: Identification of mutations in the AIPL1, CRB1, GUCY2D, RPE65, and RPGRIP1 genes in patients with juvenile retinitis pigmentosa. J Med Genet 42: e67, 2005.

16. Beryozkin A, Zelinger L, Bandah-Rozenfeld D, Harel A, Strom TA, Merin S, Chowers I, Banin E and Sharon D: Mutations in CRB1 are a relatively common cause of autosomal recessive early-onset retinal degeneration in the Israeli and Palestinian populations. Invest Ophthalmol Vis Sci 54: 2068-2075, 2013.

17. Chen Y, Lin Y, Vithana EN, Jia L, Zuo X, Wong TY, Chen LJ, Zhu X, Tam PO, Gong B, et al: Common variants near ABCA1 and in PMM2 are associated with primary open-angle glaucoma. Nat Genet 46: 1115-1119, 2014.

18. Richard M, Roepman R, Aartsen WM, van Rossum AG, den Hollander AI, Knust E, Wijnholds J and Cremers FP: Towards understanding CRUMBS function in retinal dystrophies. Hum Mol Genet 15: R235-R243, 2006.

19. den Hollander AI, Johnson K, de Kok YJ, Klebes A, Brunner HG, Knust E and Cremers FP: CRB1 has a cytoplasmic domain that is functionally conserved between human and Drosophila. Hum Mol Genet 10: 2767-2773, 2001.

20. Tepass U: Crumbs, a component of the apical membrane, is required for zonula adherens formation in primary epithelia of Drosophila. Dev Biol 177: 217-225, 1996.

21. Pellikka M, Tanentzapf G, Pinto M, Smith C, McGlade CJ, Ready DF and Tepass U: Crumbs, the Drosophila homologue of human CRB1/RP12, is essential for photoreceptor morphogenesis. Nature 416: 143-149, 2002.

22. Beheshtian M, Saee Rad S, Babanejad M, Mohseni M, Hashemi H, Eshghabadi A, Hajizadeh F, Akbari MR, Kahrizi K, Riazi Esfahani M and Najmabadi H: Impact of whole exome sequencing among Iranian patients with autosomal recessive retinitis pigmentosa. Arch Iran Med 18: 776-785, 2015.

23. Thompson DA, Janecke AR, Lange J, Feathers KL, Hübner CA, McHenry CL, Stockton DW, Rammesmayer G, Lupski JR, Antinolo G, et al: Retinal degeneration associated with RDH12 mutations results from decreased 11-cis retinal synthesis due to disruption of the visual cycle. Hum Mol Genet 14: 3865-3875, 2005.

24. Bujakowska K, Audo I, Mohand-Saïd S, Lancelot ME, Antonio A, Germain A, Léveillard T, Letexier M, Saraiva JP, Lonjou C, et al: CRB1 mutations in inherited retinal dystrophies. Hum Mutat 33: 306-315, 2012

25. Mehalow AK, Kameya S, Smith RS, Hawes NL, Denegre JM, Young JA, Bechtold L, Haider NB, Tepass U, Heckenlively JR, et al: CRB1 is essential for external limiting membrane integrity and photoreceptor morphogenesis in the mammalian retina. Hum Mol Genet 12: 2179-2189, 2003.

26. Zhou Y, Tao S, Chen H, Huang L, Zhu X,Li Y, Wang Z, Lin H, Hao F, Yang Z, et al: Exome sequencing analysis identifies compound heterozygous mutation in ABCA4 in a Chinese family with Stargardt disease. PLoS One 9: e91962, 2014.

27. Wang H, Wang X, Zou X, Xu S, Li H, Soens ZT, Wang K, Li Y, Dong F, Chen R and Sui R: Comprehensive molecular diagnosis of a large Chinese Leber congenital Amaurosis cohort. Invest Ophthalmol Vis Sci 56: 3642-3655, 2015. 